\title{
FEATURE TRANSFORMATION AND SIMULATION OF SHORT TERM PRICE VARIABILITY IN REINFORCEMENT LEARNING FOR PORTFOLIO MANAGEMENT
}

\author{
Yen-Chih Lin \\ Jeremy Blum \\ Department of Computer Science and Mathematics \\ Pennsylvania State University, Harrisburg \\ 777 W. Harrisburg Pike \\ Middletown, PA, USA \\ $\{$ yq15869, jjb24\}@psu.edu
}

\begin{abstract}
Reinforcement learning has been shown capable of learning optimal strategies from imperfect information environments in order to create robust decision support systems. This paper shows that two automatic feature transformation techniques - Bayesian recurrent neural network (BRNN) for modelling future price trends and Generative Adversarial Networks (GANs) for modelling short-term realistic price variability are able to improve the performance of reinforcement learning agents in solving portfolio management problem effectively, when measured in terms of increasing profitability and reducing risks.
\end{abstract}

Keywords: Feature Transformation, Reinforcement Learning, Bayesian Neural Networks, Generative Adversarial Networks

\section{INTRODUCTION}

Portfolio management for financial markets remains a difficult problem to develop automated systems to solve. In the problem, one is given a fixed amount of funds to allocate among various instruments in a market, and one must make decision at each timestamp to maximize return while minimizing risk. Due to the complexity of potential factors that affect the price movement, it is very difficult to find a strategy that generalizes well on the many possible types of price movements.

Deep reinforcement learning models have been proven to be able to solve complex strategic problems based on imperfect information due to partially observed environments (Vinyals et al. 2017). In many such problems, the inputs of the environments are deterministic, and, as such, there are often optimal strategies that an agent can take based on input data. In financial markets, on the other hand, due to their stochastic nature, signals for optimal strategies can be very sparse, hence it can be very difficult to train a wellperforming agent. Previous research has shown that with carefully designed training approaches, recurrent reinforcement learning algorithms can learn profitable trading strategies that generate market returns better than market index (Deng el al. 2017), which is commonly used as a baseline for trading in financial markets. In the previous research, however, only price data, e.g. open, close, high, and low, are used, and only a few well-known stock indices are used for training and testing which may be prone to bias. 


\section{Lin and Blum}

The performance of a reinforcement learning agent in this paper is measured by the profitability and risk. Profitability and risk are represented as mean and standard deviation of final returns, respectively. Final returns are generated by testing the agent with a number of randomly selected future unseen periods.

Specifically, this paper demonstrates that two automatic feature transformation techniques can increase the performance of the agent with low pre-processing overhead. The first technique uses Bayesian neural networks (BNN) to model probability distributions of future prices. Due to the stochastic nature of financial market data, neural networks are prone to overfitting the training data. Bayesian regularization has been shown to be suitable for this type of data (Ticknor 2013). In this paper, a larger BNN containing convolution and recurrent layers is used, rather than three layers of perceptrons. This additional complexity enables our approach to model hourly rather than daily price changes, and extend the prediction horizon further into future, predicting five to ten intervals ahead rather than just one. In addition, this work includes the use of generative adversarial networks (GAN) to simulate repetitive, short-term patterns in the financial market data. Our approach uses the generator to generate simulated price data with different long term trends to prevent the agent from overfitting long term and non-repetitive patterns.

\section{RELATED WORK}

Significant recent research has applied reinforcement learning to the portfolio management problem. Recurrent reinforcement learning (RRL), in particular, has been successfully applied, due to the ability of recurrent networks to provide better representation of time-series data than multi-layer perceptrons (Hochreiter and Schmidhuber 1997). Similarly, direct reinforcement learning (DRL) has been used to train the reinforcement agents under risk-adjusted constraint where the Sharpe ratio is included as consideration during training (Moody and Saffell 2001).

In addition to only using recurrent network as policy network to interpret data directly, other research has used deep fully connected networks and convolution neural networks (CNN) as feature transformation to improve agent performance (Deng et al. 2017, Jiang and Liang 2017). In such feature transformation networks, training loss produced by the policy network is propagated directly to the feature transformation network such that it can adapt its learned features to help the agent learn better strategies.

Trading strategies that rely only on long-term trends may suffer from short-term loss. Some approaches, such as using stacked dynamic recurrent reinforcement learning, try to solve the challenges that arise when long-term future rewards are hard to propagate back to past actions taken by an agent (Aboussalah and Lee 2020).

Another advantage of using reinforcement learning for the portfolio management problem is that agent behavior can be guided by adjusting the objective function of the policy network. Almahdi and Yang (2017) proposed a new risk-management objective function, Expected Maximum Drawdown, instead of other commonly used objective functions, e.g. Sharpe ratio and Calmar ratio. The authors showed that the proposed objective function is significantly better than the other two in terms of max return and max drawdown.

Approaches to price and trend forecasting have evolved over time. Prior to the widespread use of deep neural networks, the Autoregressive Integrated Moving Average (ARIMA) model was the most commonly used method in price forecasting (Adebiyi, Adewumi, and Ayo 2014). More recent forecasting models have applied deep neural networks to this problem. For example, probabilistic neural networks use Bayesian regularization to alleviate the overfitting problem, e.g. (Ticknor 2013). Various forms of recurrent neural network, including Long-Short Term Memory (LSTM), have been applied to the portfolio management problem, e.g. (Lin, Yang, and Song 2019) and (Yoshihara et al. 2014). The incorporation of unstructured data, such as news data, into the abstract representation to improve the predictive power of recurrent networks, e.g. (Hu et al. 2017) and (Hagenau, Liebmann, and Neumann 2013). Finally, given the large data requirements of reinforcement learning, Generative Adversarial Networks (GANs) have been used to generate additional training data for these approaches, e.g. (Zhang et al. 2019) and (Feng et al. 2019). 


\section{Lin and Blum}

In this paper, we extend approaches in (Ticknor 2013), (Hu et al. 2017), and (Feng et al. 2019) through the formulation of novel feature transformation networks. In addition, we extend approaches in (Zhang et al. 2019) to apply GAN to generate realistic simulations of short-term price fluctuations.

\section{METHODOLOGY}

At a high level, our proposed approach contains three distinct training stages. In the first stage, a Bayesian neural network is trained to predict future prices. In the second, a GAN is trained to generate simulated financial market data, which aims to model short-term patterns while avoiding modeling long-term patterns. In the final stage, a reinforcement learning agent is trained to make portfolio management decisions using different sets of input features. Feature transformation approaches are evaluated based on the effectiveness of increasing agent's performance compared to a baseline approach.

The evaluation of the approaches is conducted using the prices of 7 cryptocurrencies as input data, BTC, ETH, NEO, LTC, IOT, EOS and XRP. The prices used are from September 2017 to October 2019. The input data, consisting of hourly prices, is processed as follows. A sliding window, of size 512 time periods, slides through the training set to generate time frames, where each frame is a training instance. The training set is created using the first $70 \%$ of the time periods of the entire data set. The price data, for training purposes, is normalized frame-by-frame by dividing each price data by the max value of the price data in the window. This normalization approach makes the models less likely to overfit by memorizing the price range of each financial instrument. Note that, the normalization is not performed by dividing the prices by the maximum value of entire price data, which would potentially cause information leakage to the training process.

\subsection{Technical Indicators}

For a baseline performance evaluation, 60 technical indicators (see appendix) are used to generate baseline features from the price data, including open, close, high, and low prices. These technical indicators are commonly used in market price technical analysis. The data generated by the technical indicators, along with price data, are used as input features to train the baseline reinforcement learning agent. These input features are also used to train the Bayesian network price prediction model, which is then compared against the baseline approach.

\subsection{Bayesian Recurrent Neural Network}

The goal of time-series forecasting is to predict the repetitive patterns that are observed from the past data. If some trend patterns are the result of a specific event that is unlikely to occur again, it would be undesirable for the model to predict that kind of pattern. This type of prediction would be indicative of overfitting on the training data, especially for stochastic time-series data such as market prices.

Indeed, one short-coming of recurrent neural networks (RNN) is that they tend to overfit stochastic timeseries data, while Bayesian neural network $(\mathrm{BNN})$ can provides effective regularization to prevent this overfitting (Ticknor 2013). In a BNN, each cell is presented as a Gaussian distribution. At each inference, the distribution is sampled multiple times and the average of the sampled values are passed as output to the next cell. In this paper, we introduce the idea of a Bayesian Recurrent Neural Network (BRNN), which extends the work of (Ticknor 2013) by adding convolutional and recurrent layers in order to produce better prediction results. Comparing to non-distributional RNN, BRNN produces a distribution for price predictions instead of producing a point predictions.

The Bayesian recurrent neural network (BRNN) is implemented using the Tensorflow probability library. It consists of five convolutional blocks, two time-distributed fully connected layers, three recurrent blocks, and two fully connected layers. Each convolutional block contains one convolutional layer, batch normalization and activation function. Average pooling, with a $2 \times 2$ window, is performed every two convolutional blocks. Each recurrent block contains one recurrent layer with long-short term memory 
(LSTM) cells, batch normalization, and an activation function. Rectified linear units (RELUs) are used as the activation functions for each convolutional block and fully connected layer while hyperbolic tangent (tanh) is used for each recurrent block. The sigmoid activation function is used at the output layer since target price data for prediction is normalized to range $[0,1]$.

The BRNN produces accurate predictions for future prices. Figure 1 show price prediction for a time horizon of eight hours. The prediction of the BRNN for prices 8 hours from now, shown in blue, track actual future prices, shown in red.

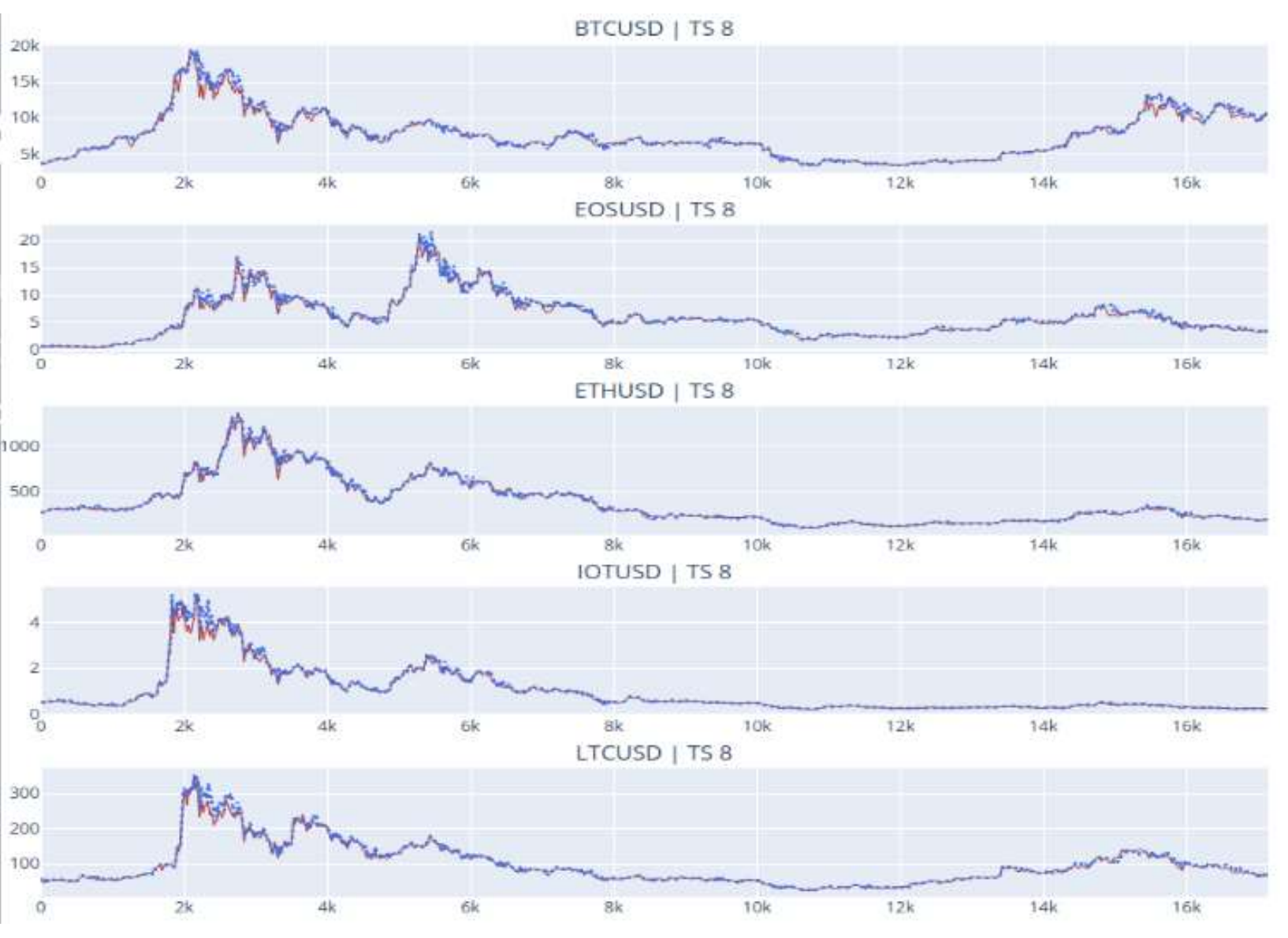

Figure 1: Price prediction of Bayesian network. The vertical axes show prices in USD. The horizontal axes display the time periods, which unit is one hour. Red lines represent actual prices while blue dash lines represents predicted prices. TS represents time steps predicted. In this chart, the BRNN is trained to predict the prices of 8 hours ahead.

\subsection{Generative Adversarial Network}

The stochasticity of financial market data comes from nonrepetitive events and long-term trends affected by multiple factors combined. Since particular price patterns are unlikely to repeat in the exactly the same manner, it is beneficial to prevent the agent from overfitting on those patterns. Our approach enables the agent to observe the sparse repetitive patterns with simulations of repetitive short-term data, produced with a generative adversarial network (GAN).

The goal of generating the simulated price data is to present short-term repetitive patterns within a variety of non-repetitive, long-term trends, so the agent will focus more on fitting the repetitive patterns. This approach can be considered as a data augmentation technique for time-series data. 
A GAN consists of a generator, which generates simulated price data, and a discriminator, which discriminates the real and simulated price data. The generator, used in this work, consists of three recurrent blocks and five convolutional blocks, while the discriminator consists of five convolutional blocks, three recurrent blocks and two fully connected layers. The output of the generator and real price data are passed to the discriminator along with the true/false labels. The discriminator needs to discriminate which is real and which is fake.

To prevent the discriminator from overfitting on the real price data, we minimize the information of longterm non-repetitive trends in each training sample. This is achieved by keeping the observation size of each sample small and normalizing each sample frame-by-frame as mentioned previously. This way, the discriminator is only able to discriminate from the normalized, short-term, repetitive patterns. As a result, the generator will be less likely to overfit on the non-repetitive patterns in order to reduce the loss. Examples of simulated prices generated by the GAN are shown in figure 2 . These figures seem to indicate that the GAN reproduces short-term volatility patterns similar to real price fluctuations.

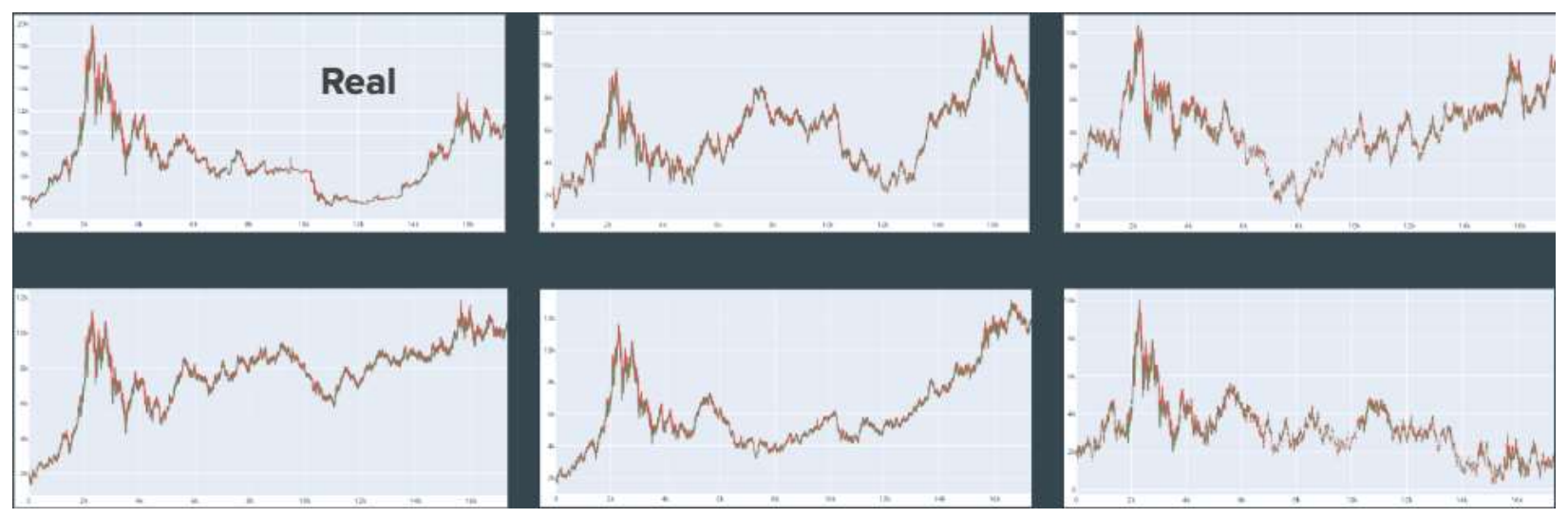

Figure 2: Top left is the real Bitcoin price from September 2017 to October 2019. The rest are the fake prices generated by the generator. The vertical axes show prices in USD. The horizontal axes display the time periods, which unit is one hour.

\subsection{Reinforcement Learning Agent}

To evaluate the effectiveness of the two proposed approaches, we implemented a feature-rich reinforcement learning environment using OpenAI's Gym. The environment provides a simulation of the trading market by taking agent's action, calculating market state, and then returning state and reward to the agent at each time step. The reward returned by the environment is the profit or loss made at the current step divided by the total asset at previous step, i.e. total asset change in percentage. In the learning environment, a transaction fee is set to $0.3 \%$ and an agent is allowed to long or short each financial instrument at each time step.

The agent follows a trading strategy uses a feature extraction network and a policy network to generate portfolio management decisions. The feature extraction network takes input data described previously, transforms the input data, and then feeds it into the policy network. The feature extraction network is trained together with the policy network. The gradient is propagated back to the feature extraction network while training the policy network, hence, these two networks can be considered as a single network.

The feature extraction network consists of either four convolutional blocks and two fully connected layers (named FE-CNN) or four fully connected layers (named FE-MLP). The policy network consists of one LSTM layer and four fully connected layers. The output of the policy network is a vector of values that sums to one which represents the asset allocation to each financial instrument in percentage. For example, if the number of trading indices is ten, the output vector will be length of ten, with values that sum to one. 
The reinforcement learning algorithm, used to train the agent in this paper, is Proximal Policy Optimization (PPO2), implemented using Stable Baselines (Schulman et al. 2017). To minimize the loss of the policy network, the agent needs to learn optimal trading strategies.

\section{RESULTS}

The effectiveness of the BRNN and GAN is evaluated as follows. A series of experiments is conducted with a parameter set that includes the feature extractor type, feature transformation methods and skipperiod. The feature extractor type can be either FE-CNN or FE-MLP. The feature transformation methods can be either baseline, Bayesian or GAN. Note that features for Bayesian type for training the agent includes features from baseline, which is the 60 technical indicators. However, the GAN feature transformation type does not include baseline features. Finally, in order to measure the influence of trading frequency on the results, we consider a skip period, or the frequency with which trades may be made. Skip periods of 3, 5 and 10 time periods are considered. For example, if the skip period is 5, the agent is forced to trade every 5 time steps, and the agent cannot respond to the price change during this period. By applying this mechanism, the agent is forced to predict trends over longer time horizons.

The features generated by BRNN and GAN are added as additional features to the input data to train the agent. The generator in GAN is used to generate either two or six times more data in additional to original price data. The first $70 \%$ of data set is used to train BRNN, GAN and the agent, and the last $30 \%$ of data set is used as evaluation set the trained reinforcement agent.

Each reinforcement agent is trained for one million steps and then tested on 20 (seeded) randomly selected time periods from the testing set. We test the agents on randomly selected short time periods instead of entire testing set at once to address the issue that a better agent should perform good and stable under any market periods. An unstable agent is not ideal for financial market trading, even if it performs very well under certain market conditions.

Each test generates 20 final asset values after trading, mean and standard deviation are then calculated from the test results. Higher mean returns represents the higher profitability of an agent, while lower standard deviation in the final asset value represents higher stability and lower risk. Since risk acceptance is subjective under different trading conditions, these two evaluation factors will not be combined to evaluate the agent. Initial funds are set to $\$ 100,000$.

At each decision point, the agent decides, for each asset, whether to short, i.e. make a bet that prices for an asset will decrease, or long, i.e. make a bet that prices will increase. In addition, the agent allocates total funds between the various assets. Figure 3, for example, shows examples of these trading actions for the IOT cryptocurrency. In the figures, red triangles represent shorting actions while green triangles represent longing actions. The numbers near the triangles represent the percentage of total asset is put into the longing/shorting actions. 

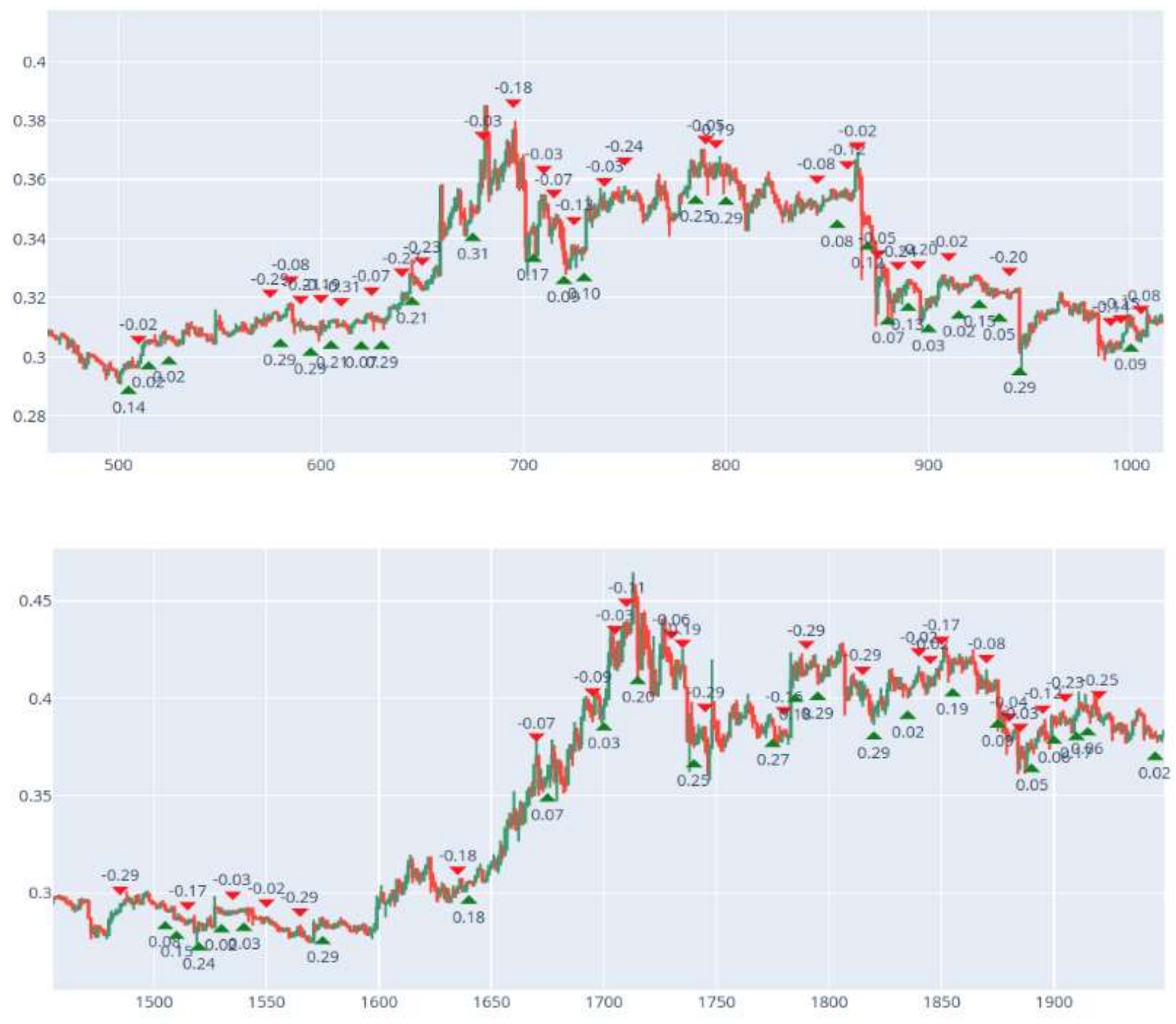

Figure 3: Sample Agent Trading Decisions. The vertical axes show prices in USD. The horizontal axes display the time periods, each of which is one hour in length.

The final experimental results under different parameter configurations can be seen in table 1 . As shown in table 1, adding future price prediction generated by BRNN always improve the performance of the agent with the same configurations in terms of both profitability and risk. 
Table 1: Test results

\begin{tabular}{|c|c|c|c|c|}
\hline $\begin{array}{l}\text { Feature } \\
\text { Extractor }\end{array}$ & $\begin{array}{c}\text { Feature } \\
\text { Transformation }\end{array}$ & $\begin{array}{l}\text { Skip } \\
\text { Period }\end{array}$ & $\begin{array}{l}\text { Mean Return } \\
\text { (Profitability) }\end{array}$ & $\begin{array}{l}\text { Standard } \\
\text { Deviation in } \\
\text { Final Value } \\
\text { (Risk) }\end{array}$ \\
\hline FE-MLP & Baseline & 3 & $-31.5 \%$ & $9.90 \%$ \\
\hline FE-MLP & Baseline+Bayesian & 3 & $12.3 \%$ & $7.01 \%$ \\
\hline FE-MLP & Baseline & 5 & $39.5 \%$ & $15.71 \%$ \\
\hline FE-MLP & Baseline+Bayesian & 5 & $60.0 \%$ & $12.99 \%$ \\
\hline FE-CNN & Baseline & 5 & $38.0 \%$ & $9.70 \%$ \\
\hline FE-CNN & Baseline+Bayesian & 5 & $62.5 \%$ & $9.26 \%$ \\
\hline FE-CNN & Baseline & 10 & $18.8 \%$ & $6.74 \%$ \\
\hline FE-CNN & Baseline+Bayesian & 10 & $37.6 \%$ & $4.63 \%$ \\
\hline FE-MLP & GAN (2x data) & 10 & $32.9 \%$ & $5.46 \%$ \\
\hline FE-MLP & GAN (6x data) & 10 & $28.4 \%$ & $4.05 \%$ \\
\hline
\end{tabular}

In tests with the GAN, on the other hand, training with simulated data reduces instability, but may also reduce the profitability. In addition, it can be seen that using FE-CNN is better than using FE-MLP. There are significantly fewer weight to learn in the convolutional networks, which are likely responsible for the improved performance of the CNN approach.

Finally, there is a tradeoff seen in varying the length of the skip periods. Lengthening the skip period time helps reduce unnecessary transactions and increase profitability effectively. However, beyond a point, the limits of predicting future price levels and limits in the ability of an agent to respond to the market changes in a timely fashion, and as a result, profitability will be adversely affected.

\section{CONCLUSIONS}

The experimental results show that the approaches introduced in this work can effectively improve the performance of the reinforcement agents for the portfolio management problem. Bayesian Recurrent Neural Networks (BRNNs) can increase the profitability of an agent more while Generative Adversarial Networks (GANs) can reduce risk. BRNNs increased final return by between $14 \%$ and $43 \%$, while reducing standard deviation by between $5 \%$ and $7 \%$. Using GANs to simulate short term price data for training the reinforcement agents, increased final return by between $8 \%$ and $11 \%$ while reducing standard deviation in the final portfolio value by between $20 \%$ and $40 \%$.

These results suggest that BRNNs provide effective mechanisms for predicting long term future price changes. GANs on the other hand, provides more diverse short-term price patterns in the training set, and as result, the agent sees more price patterns under different scenarios, which greatly increases the generalization of the strategies learned by the agent.

\section{APPENDIX A}

Technical indicators are implemented using Python Financial Technical Analysis (FinTA) library, and include: ADX, AO, APZ, ATR, BASP, BASPN, BBANDS, BBWIDTH, CCI, CFI, CHAIKIN, CHANDELIER, CMO, COPP, DEMA, DMI, EBBP, EFI, EMA, ER, FISH, FVE, HMA, ICHIMOKU, 
IFTRSI, KAMA, KC, KST, MACD, MFI, MOM, PERCENTB, PPO, ROC, RSI, SAR, SMA, SMM, SMMA, SQZMI, STOCH, STOCHD, STOCHRSI, TEMA, TP, TR, TRIMA, TRIX, TSI, UO, VFI, VAMA, VWAP, VWMACD, VZO, WILLIAMS, WMA, WOBV, WTO, and ZLEMA.

\section{REFERENCES}

A. Adebiyi, A. Adewumi, and C. Ayo, "Stock price prediction using the arima model," Mar. 2014. DOI: 10. 1109/UKSim.2014.67.

A. M. Aboussalah and C.-G. Lee, "Continuous control with stacked deep dynamic recurrent reinforcement learning for portfolio optimization," Expert Systems with Applications, vol. 140, p. $112891,2020$.

S. Almahdi and S. Y. Yang, "An adaptive portfolio trading system: A risk-return portfolio optimization using recurrent reinforcement learning with expected maximum drawdown," Expert Systems with Applications, vol. 87, pp. 267-279, 2017.

Y. Deng, F. Bao, Y. Kong, Z. Ren, and Q. Dai, “Deep direct reinforcement learning for financial signal representation and trading," IEEE Transactions on Neural Networks and Learning Systems, vol. 28, no. 3, pp. 653-64, Mar. 2017. DOI: 10.1109/TNNLS.2016.2522401.

F. Feng, H. Chen, X. He, J. Ding, M. Sun, and T.-S. Chua, "Enhancing stock movement prediction with adversarial training," in Proceedings of the Twenty-Eighth International Joint Conference on Artificial Intelligence, IJCAI-19, International Joint Conferences on Artificial Intelligence Organization, Jul. 2019, pp. 5843-5849.

M. Hagenau, M. Liebmann, and D. Neumann, "Automated news reading: Stock price prediction based on financial news using context-capturing features," Decision Support Systems, vol. 55, no. 3, pp. 685697, 2013.

S. Hochreiter and J. Schmidhuber, "Long short-term memory," Neural Comput., vol. 9, no. 8, pp. 17351780, Nov. 1997.

Z. Hu, W. Liu, J. Bian, X. Liu, and T.-Y. Liu, "Listening to chaotic whispers: A deep learning framework for news-oriented stock trend prediction," Dec. 2017.

Z. Jiang and J. Liang, "Cryptocurrency portfolio management with deep reinforcement learning," in 2017 Intelligent Systems Conference (IntelliSys), Sep. 2017, pp. 905-913.

X. Lin, Z. Yang, and Y. Song, "Short-term stock price prediction based on echo state networks," Expert Systems with Applications, vol. 36, no. 3, Part 2, pp. 7313-7317, 2009.

J. Moody and M. Saffell, "Learning to trade via direct reinforcement," IEEE Transactions on Neural Networks, vol. 12, no. 4, pp. 875-889, Jul. 2001.

J. Schulman, F. Wolski, P. Dhariwal, A. Radford, and O. Klimov, "Proximal policy optimization algorithms," Jul. 2017.

J. Ticknor, "A bayesian regularized artificial neural network for stock market forecasting," Expert Systems with Applications, vol. 40, pp. 5501-5506, Oct. 2013.

O. Vinyals, T. Ewalds, S. Bartunov, P. Georgiev, A. Vezhnevets, M. Yeo, A. Makhzani, H. K"uttler, J. Agapiou, J. Schrittwieser, J. Quan, S. Gaffney, S. Petersen, K. Simonyan, T. Schaul, H. Van Hasselt, D. Silver, T. Lillicrap, K. Calderone, and R. Tsing, "Starcraft ii: A new challenge for reinforcement learning," Aug. 2017.

A. Yoshihara, K. Fujikawa, K. Seki, and K. Uehara, "Predicting stock market trends by recurrent deep neural networks," vol. 8862, Dec. 2014, pp. 759-769.

K. Zhang, G. Zhong, J. Dong, S. Wang, and Y. Wang, "Stock market prediction based on generative adversarial network," Procedia Computer Science, vol. 147, pp. 400-406, 2019, 2018 International Conference on Identification, Information and Knowledge in the Internet of Things. 


\section{AUTHOR BIOGRAPHIES}

YEN-CHIH LIN holds an M.S. in Computer Science from the Pennsylvania State University. His email address is yq15869@psu.edu.

JEREMY J. BLUM is an Associate Professor of Computer Science at the Pennsylvania State University, Harrisburg. He holds a D.Sc. in Computer Science and M.S. in Computational Sciences from the George Washington University, and a B.A. in Economics from Washington University in St. Louis. His email address is jjb24@psu.edu. 